\title{
A comparison of SNPs and microsatellites as linkage mapping markers: lessons from the zebra finch (Taeniopygia guttata)
}

\author{
Alexander D Ball*, Jessica Stapley, Deborah A Dawson, Tim R Birkhead, Terry Burke and Jon Slate
}

\begin{abstract}
Background: Genetic linkage maps are essential tools when searching for quantitative trait loci (QTL). To maximize genome coverage and provide an evenly spaced marker distribution a combination of different types of genetic marker are sometimes used. In this study we created linkage maps of four zebra finch (Taeniopygia guttata) chromosomes (1, 1A, 2 and 9) using two types of marker, Single Nucleotide Polymorphisms (SNPs) and microsatellites. To assess the effectiveness and accuracy of each kind of marker we compared maps built with each marker type separately and with both types of marker combined. Linkage map marker order was validated by making comparisons to the assembled zebra finch genome sequence.
\end{abstract}

Results: We showed that marker order was less reliable and linkage map lengths were inflated for microsatellite maps relative to SNP maps, apparently due to differing error rates between the two types of marker. Guidelines on how to minimise the effects of error are provided. In particular, we show that when combining both types of marker the conventional process of building linkage maps, whereby the most informative markers are added to the map first, has to be modified in order to improve map accuracy.

Conclusions: When using multiple types and large numbers of markers to create dense linkage maps, the least error prone loci (SNPs) rather than the most informative should be used to create framework maps before the addition of other potentially more error prone markers (microsatellites). This raises questions about the accuracy of marker order and predicted recombination rates in previous microsatellite linkage maps which were created using the conventional building process, however, provided suitable error detection strategies are followed microsatellite-based maps can continue to be regarded as reasonably reliable.

\section{Background}

Linkage maps are fundamental tools in many genetic studies and have been created using various types of polymorphic markers since their conception by Sturtevant in 1913 [1]. Genetic linkage maps determine the linear position of genes or markers on a chromosome. They also provide information on genome wide recombination rates, as well as insight into intra and inter-species gene rearrangements within and between chromosomes; thus maps are useful in the study of evolutionary and comparative genomics [2-4]. However one of their most important applications is in the search for Mendelian and

\footnotetext{
* Correspondence: a.d.ball@sheffield.ac.uk

${ }^{1}$ Department of Animal and Plant Sciences, University of Sheffield, Sheffield, S10 2TN, UK

Full list of author information is available at the end of the article
}

Quantitative Trait Loci (QTL) [5,6]. Over the last four decades advances in molecular technology have meant a wider range and greater number of genetic markers have become available, enabling linkage maps to be created for an increasing number of species, including many nonmodel organisms [7-9]. It is therefore timely to consider how different markers and different building approaches may influence the accuracy of genetic maps.

Two key components to consider when constructing a linkage map are the number and type of markers to use. Phenotypic (i.e. visible) markers were the first to be utilized, but now the choice has been extended to include a range of molecular markers including allozymes, Random Amplified Polymorphic DNA (RAPDs), Restriction Fragment Length Polymorphisms (RFLPs), Amplified Fragment Length Polymorphisms (AFLPs), Sequence-Tagged 
Sites (STSs), microsatellites (Simple Sequence Repeats) and SNPs. Each of these exhibit slightly different advantages and disadvantages but there are three main considerations when deciding which genetic markers to use in linkage map construction: (i) the markers need to be polymorphic, (ii) they need to be evenly spread across the genome or region of interest and provide dense marker coverage, and (iii) they must have a low genotyping error rate.

Microsatellites are obvious candidates for linkage mapping; they are highly polymorphic, relatively easy and cheap to score (once a library is established), and can exhibit cross-species utility in closely related species [1013]. As a result they have been widely used in linkage mapping studies of humans, model organisms, agriculturally-important organisms and wild vertebrate populations [14-20]. Recently though, technological advances in locating and genotyping Single Nucleotide Polymorphisms (SNPs) has led to a decrease in both discovery time and genotyping cost $[21,22]$. This has caused an increase in their application in linkage mapping studies, exemplified in an updated high density chicken linkage map with 8599 SNPs [23] compared to the earlier map of 1889 molecular markers of which the majority were microsatellites [24]. This increased use of SNPs comes despite their (usually) biallelic nature, which means they provide relatively less information per locus. Lower variability means that they reveal fewer informative meioses, making linkage between markers harder to detect. To combat this, an increased number of markers, which are evenly spaced and cover a high proportion of the genome, can be used [25]. Additionally, it is possible to build maps with a combination of low-density, high-variability microsatellites and high-density, low-variability SNPs. The rationale of this approach is that the microsatellites might act as anchors and cause otherwise unassigned SNPs to be assimilated into linkage groups. This would produce linkage maps of greater accuracy or density, which will then be better suited to searching for QTL.

Microsatellites and SNPs also differ with respect to consideration (iii); the error rate. Markers with lower error rates obviously produce more accurate linkage data. A trade-off associated with the highly polymorphic nature of microsatellites is that they can have relatively high genotyping error rates [26,27]. Methods of genotyping and allele-calling microsatellites are only semi-automated, which can introduce human-based errors. Modern SNP genotyping platforms are almost fully automated and error rates tend to be much lower [28]. In previous studies much emphasis has been placed on identifying the consequences of genotyping error on the accuracy of population genetic analyses [29,30]. It has been acknowledged that these errors can impact on parentage inference or population structure analyses
$[26,27,31]$, but only recently has attention been focused on the extent to which genotyping errors can impact genetic linkage mapping [32,33]. In the human genetics literature, the relative merits of SNPs and microsatellite for genome-wide scans of complex diseases have been compared [34-38], but these studies have focused on the mapping of causative loci rather than the necessary initial step of map construction.

In a recent simulation study, where markers were compared for mapping accuracy, a difference in power between bi-allelic markers and polymorphic markers was reported, with a higher density of SNPs required to accurately produce similar results to the polymorphic microsatellites [32]. However, it was shown that when genotyping error rates are low, both SNP and microsatellite maps can be produced accurately. When a simulated $5 \%$ genotyping error rate was introduced to microsatellite genotyping, map inflation (sometimes by over 50\%), incorrect marker order and even occasional allocation of markers to the incorrect linkage group occurred. This 5\% level of error is not unprecedented in microsatellite genotyping, even when using invasive techniques of DNA acquisition $[27,39]$. Clearly there is a need to eliminate markers with genotyping errors $\sim 5 \%$ wherever possible before proceeding with linkage analysis.

The aim of this study was to compare linkage maps built with microsatellites, SNPs and a combination of both markers to determine the ability of each method to produce accurate linkage maps. A linkage map of the zebra finch (Taeniopygia guttata) genome using SNP markers has recently been released [40], and a genome assembly is publicly available, which facilitates comparisons of marker order between linkage maps and physical assemblies. By independently constructing microsatellite linkage maps of the zebra finch macro-chromosomes 1 , $1 \mathrm{~A}, 2$ and 9 , using the same pedigree as was used in the SNP map, we were able to explore how marker type influenced map length and order. Guidelines for mapping studies that employ microsatellites and SNPs simultaneously are provided, as we recognise that sometimes it is practical to combine datasets containing both types of marker.

\section{Methods}

The captive zebra finch population used in this study is one which has been maintained within the Department of Animal and Plant Sciences at the University of Sheffield since 1985 under the stewardship of TRB. The 3-generation mapping pedigree comprises 354 individuals and is the same pedigree as that used to build a SNP map of the whole zebra finch genome [40]. Genomic DNA was extracted from either blood or tissue samples using the ammonium acetate precipitation method [41]. The maintenance of the zebra finch pedigree along with the extrac- 
tion of DNA was carried out in line with the UK Home Office guidelines under project license number 40/2788.

\section{SNP discovery and genotyping}

The SNP genotyping data used in this study is the same as that previously published in [40]. This previous study was the first genome-wide zebra finch linkage map and incorporated 876 SNPs across 45 linkage groups. On the four chromosomes which are compared in our study there are 250 SNPs (73 on Tgu1, 71 on Tgu1A, 82 on Tgu2 and 24 on Tgu9). The SNPs were identified from cDNA sequences generated by Washington University Genome Researching Centre on a 454 Life Sciences ultra-high throughput pyrosequencing platform. The genotyping was undertaken by Illumina (San Diego) using the golden gate platform where 1356 potential SNPs were analysed. The genotyping data was estimated to be $100 \%$ reproducible, had a $95.5 \%$ call rate and a $0.17 \%$ parent-offspring error rate. After false and monomorphic SNPs and SNPs with a minor allele frequency $<0.05$ were discarded, 876 SNPs remained and were assembled into linkage groups. For a more detailed account of the SNP genotyping see [40].

\section{Microsatellite discovery}

In order to discover polymorphic microsatellites, three methods were implemented. Initially, previously published microsatellites from the avian literature and a set of highly conserved microsatellite loci located in the zebra finch expressed sequence tag (EST) public databases were assessed $[42,43]$. By predicting chromosomal locations of the loci in the chicken genome sequence assembly [44], we discovered that the chromosomes 1, 2 and 9 had the highest number of already available microsatellites and so we subsequently focused on locating more microsatellites within these three chromosomes. However, in the zebra finch this equates to 4 chromosomes because Chicken chromosome 1 shares homology with two chromosomes in passerine birds $[2,40,45,46]$. Thus we were focusing on 4 chromosomes in the zebra finch (Tgu1, Tgu1A, Tgu2 and Tgu9).

We attempted to increase our marker density on these 4 chromosomes using the program SPUTNIK (Sputnik http://abajian.net/sputnik/) to search two zebra finch sequence databases for microsatellites. The first approach took advantage of the zebra finch EST sequences located on the National Centre for Biotechnology Information (NCBI) website (described in [47]), and the second searched the zebra finch Whole Genome Shotgun (WGS) GenBank tracefiles, as the assembled zebra finch genome was unavailable at that point. These searches were restricted to dinucleotide repeat motifs. In general, microsatellites with uninterrupted long repeats have been shown to be the most variable $[48,49]$. Therefore, only repeats of greater than $40 \mathrm{bp}$ and with at least $90 \%$ purity were considered for further use. This ensured that microsatellites with the greatest potential for high variability were selected. As there can be more than one trace file for any region of the genome, files containing overlapping sequence were combined into contigs using the CAP3 contig assembly program [50].

A zebra finch homologous location for each microsatellite marker was found by directly comparing its sequence against the zebra finch genome assembly. Chromosomal locations were identified by Basic Local Alignment Search Tool (BLAST) analysis to compare the avian microsatellite or the zebra finch sequence (Whole Genome Shotgun (WGS) tracefiles or ESTs) against the zebra finch genome sequence (version 1, Zebra Finch Genome Consortium). BLAST analyses were carried out using Standalone BLAST version 2.2.17 [51] using the default parameters with minor modifications $(\mathrm{W}=10, \mathrm{~m}=8, \mathrm{e}=$ $\left.1 \mathrm{e}^{-5}\right)$. The chromosome position with the lowest e-value was considered a good hit if the e-value of the hit was less than $1 \mathrm{e}^{-10}$ and the difference between it and the next best hit was 10 decimal places. In general, the length of the query sequence significantly affects whether a reliable BLAST hit can be found [3]. However, the zebra finch WGS sequence trace files and ESTs were usually about $600 \mathrm{bp}$ long and thus produced reliable hits on the genome with e-values of much less than 1e-5 (See additional file 1: Table S1, for characterization of the microsatellites). These positions allowed us to focus our attention on the loci located only on the chromosomes of interest (Tgu1, Tgu1A, Tgu2 and Tgu9).

\section{Microsatellite primer design and testing}

There are three minor distinctions between the way loci were identified and primers designed in this study. These can be discerned by the names used for each primer pair, with the prefixes ZF, TG and ZEST used to discriminate between the different design methods (Table 1).

First, to increase the probability of microsatellite coamplification in other species, consensus sequences were used to identify the most conserved regions for primer design. For these markers, prefixed "TG", the primer sites are in regions of $100 \%$ identity between the zebra finch and chicken (their design is described elsewhere [43]). Second, primers for markers with prefix "ZF" were created using only the zebra finch sequence from the WGS sequence trace files. Finally, markers labeled ZEST were designed from sequences first reported in [47] and are microsatellites isolated from the zebra finch EST Genbank entries. Primers were designed using these zebra finch EST sequences rather than genomic sequence.

Primers were designed using the web interface of the program PRIMER3 ([52], http://frodo.wi.mit.edu/ primer3/). The PRIMER3 default parameters were used, 
Table 1: Summary of genotyping results.

\begin{tabular}{|c|c|c|c|c|c|c|c|c|c|c|}
\hline Locus & $\begin{array}{l}\text { Locus } \\
\text { reference }\end{array}$ & $\begin{array}{l}\text { Number of } \\
\text { individuals } \\
\text { genotyped } \\
\text { in study } \\
\text { population }\end{array}$ & $\begin{array}{l}\text { Predicted } \\
\text { allele size }\end{array}$ & $\begin{array}{l}\text { Observed } \\
\text { allele size } \\
\text { range }\end{array}$ & $\begin{array}{l}\text { Number } \\
\text { of alleles } \\
\text { obs. }\end{array}$ & $\mathrm{He}$ & Ho & $\begin{array}{l}\text { Est. null } \\
\text { allele } \\
\text { freq. } \\
\text { (CERVUS } \\
\text { v3.0) }\end{array}$ & $\begin{array}{l}\text { Mean } \\
\text { estimate } \\
\text { d error } \\
\text { rate } \\
\text { (CERVUS } \\
\text { v3.0) }\end{array}$ & $\begin{array}{l}\text { Multiplex } \\
\text { set }\end{array}$ \\
\hline ZF01-020 & This study & 320 & 178 & $160-261$ & 15 & 0.69 & 0.59 & 0.075 & 0.007 & 3 \\
\hline ZF01-025 & This study & 334 & 211 & $188-217$ & 11 & 0.78 & 0.77 & 0.005 & 0 & 2,3 \\
\hline TG01-040 & This study & 307 & 286 & $286-294$ & 7 & 0.69 & 0.70 & -0.011 & 0 & 2 \\
\hline ZF01-054a & This study & 308 & 185 & 157-188 & 13 & 0.89 & 0.75 & 0.083 & 0.002 & 2 \\
\hline ZF01-139b & This study & 330 & 188 & $156-209$ & 4 & 0.53 & 0.55 & -0.007 & 0.040 & 4 \\
\hline ZF01-136 & This study & 299 & 367 & $333-373$ & 10 & 0.73 & 0.55 & $0.138^{\$}$ & 0 & 2 \\
\hline ZF01-170c & [47] & 21 & 316 & $257-305$ & 9 & 0.83 & 0.86 & -0.032 & - & 4 \\
\hline ZF01-161 & This study & 333 & 159 & $125-168$ & 8 & 0.70 & 0.74 & -0.036 & 0 & 4 \\
\hline ZF01-190 & This study & 329 & 342 & $308-366$ & 12 & 0.83 & 0.87 & -0.026 & 0 & 3 \\
\hline ZF01-196 & This study & 334 & 292 & $270-304$ & 7 & 0.74 & 0.77 & -0.014 & 0 & $2,3,4$ \\
\hline Tgu12 & This study & 331 & 282 & $248-273$ & 10 & 0.79 & 0.75 & 0.026 & 0.016 & 3 \\
\hline ZF01-180b & [43] & 331 & 213 & $166-218$ & 8 & 0.76 & 0.66 & 0.071 & 0.060 & 4 \\
\hline ZF01-081b & This study & 337 & 233 & $130-154$ & 5 & 0.55 & 0.51 & 0.031 & 0.077 & 4 \\
\hline ZF02-068 & This study & 292 & 185 & $121-152$ & 7 & 0.70 & 0.50 & $0.157^{\$}$ & 0.010 & 2 \\
\hline ZF02-038 & This study & 301 & 233 & $211-245$ & 11 & 0.77 & 0.66 & 0.074 & 0.085 & 2 \\
\hline TG02-078 & [43] & 314 & 309 & $308-321$ & 6 & 0.73 & 0.76 & -0.024 & 0 & 1 \\
\hline TG02-088 & [43] & 310 & 268 & $263-269$ & 5 & 0.73 & 0.77 & -0.034 & 0 & 1 \\
\hline Ase44 & [64] & 303 & 268 & $308-329$ & 8 & 0.81 & 0.79 & 0.010 & 0.006 & 2 \\
\hline ZF02-128 & This study & 301 & 374 & $365-435$ & 9 & 0.83 & 0.79 & 0.022 & 0.005 & 2 \\
\hline ZF02-129 & This study & 329 & 169 & $124-172$ & 11 & 0.83 & 0.82 & 0.004 & 0.005 & 3 \\
\hline $\begin{array}{l}\text { ZEST09- } \\
005\end{array}$ & $\begin{array}{l}\text { Dawson et } \\
\text { al. } \\
\text { unpublished }\end{array}$ & 316 & 168 & $155-165$ & 5 & 0.74 & 0.67 & 0.047 & 0 & 1 \\
\hline Smm4 & [65] & 313 & 332 & $332-341$ & 6 & 0.57 & 0.50 & $0.049 \$$ & 0.018 & 1 \\
\hline Cpi7 & {$[66]$} & 308 & 128 & 119-131 & 5 & 0.54 & 0.55 & -0.016 & 0 & 1 \\
\hline $\begin{array}{l}\text { ZEST09- } \\
018\end{array}$ & $\begin{array}{l}\text { Dawson et } \\
\text { al. } \\
\text { unpublished }\end{array}$ & 289 & 285 & $282-293$ & 8 & 0.70 & 0.75 & -0.039 & 0 & 1 \\
\hline $\begin{array}{l}\text { ZEST09- } \\
021\end{array}$ & $\begin{array}{l}\text { Dawson et } \\
\text { al. } \\
\text { unpublished }\end{array}$ & 311 & 119 & $114-121$ & 5 & 0.47 & 0.41 & 0.096 & 0 & 1 \\
\hline $\begin{array}{l}\text { ZEST09- } \\
025\end{array}$ & $\begin{array}{l}\text { Dawson et } \\
\text { al. } \\
\text { unpublished }\end{array}$ & 281 & 167 & $164-170$ & 6 & 0.71 & 0.69 & 0.014 & 0 & 1 \\
\hline
\end{tabular}

Information is for the 26 zebra finch (Taeniopygia guttata) microsatellite markers used to create the linkage maps for the zebra finch chromosomes 1, 1A, 2 and 9. See additional file 1: Table S1 for more information.

$\mathrm{H}_{\mathrm{e}}, \mathrm{H}_{\mathrm{o}}$ expected and observed heterozygosity (calculated using CERVUS v3.0)

$\$$, Markers with null alleles; null alleles were detectable by following their segregation through the pedigree. Null alleles were rescored as allele 99 before running through CriMap. Null allele frequencies are calculated using the original genotypes and are based on the excess of homozygous individuals.

a, Excluded after CHROMPIC revealed an excess of double recombination events with adjacent markers, indicative of high error rate.

$b$, Excluded from linkage maps as parent-offspring mismatches estimated the error rate $>0.02$

c, Could not be accurately scored after multiplexing. 
except that a CG clamp was required at the 3 ' end of each primer and the maximum consecutive number of the same bases (Max-polyX) was lowered to 3 . The maximum primer melting temperature $(\mathrm{Tm})$ was set at $60^{\circ} \mathrm{C}$, the minimum was set at $54^{\circ} \mathrm{C}$ and the maximum Tm difference between the forward and reverse primer was lowered to $0.5^{\circ} \mathrm{C}$. By using the settings stated above we attempted to ensure all primers would amplify with a Polymerase Chain Reaction (PCR) annealing temperature of $56^{\circ} \mathrm{C}$

The forward primer was labeled with a fluoro-dye (6FAM, 5HEX or NED) to enable allele size assignment on an ABI 3730 DNA Analyzer. The initial tests for amplification were each carried out in $10 \mu \mathrm{l}$ PCR reactions. These contained about $50 \mathrm{ng}$ of genomic DNA, $1.0 \mu \mathrm{M}$ of each primer and 0.25 units Taq DNA polymerase (ThermoprimePlus, Advanced Biotechnologies) in the manufacturer's buffer (final concentrations $20 \mathrm{mM}\left(\mathrm{NH}_{4}\right)_{2} \mathrm{SO}_{4}$, $75 \mathrm{mM}$ Tris- $\mathrm{HCl} \mathrm{pH} \mathrm{9.0,0.01 \%} \mathrm{(w/v)} \mathrm{Tween),} \mathrm{including}$ $2.0 \mathrm{mM} \mathrm{MgCl}$ and $0.2 \mathrm{mM}$ of each dNTP. PCR amplification was performed in a TETRAD DNA Engine (MJ Research, Biorad) or a Touchdown thermal cycler (Hybaid). The following PCR program was used for these singleplex test reactions, 3 minutes at $94^{\circ} \mathrm{C}$, followed by 35 cycles of 30 seconds at $94^{\circ} \mathrm{C}, 30$ seconds at $56^{\circ} \mathrm{C}$ and 30 seconds at $72^{\circ} \mathrm{C}$, then 10 minutes at $72^{\circ} \mathrm{C}$. PCR products were then separated and visualized using an ABI 3730 DNA Analyzer (Applied Biosystems) using Prism set DS30 and the ROX size standard (ABI, Foster City, USA) and allele sizes assigned using GENEMAPPER 3.7 (Applied Biosystems).

Sixty-four microsatellite primers were initially tested for PCR amplification success and polymorphism. Of these, 26 were first tested on 12 zebra finch individuals (4 wild zebra finch and 8 mapping pedigree individuals). Any markers which had more than 2 alleles were then tested on 24 birds in the mapping population. However, the 38 primers with prefix "ZF" were initially tested in all 24 individuals. After this, observed and expected heterozygosity and predicted null allele frequencies were estimated using CERVUS v3.0 [53]. A total of 26 markers displayed more than 3 alleles and had an observed heterozygosity greater than 0.3 , and were then used for further analysis. The zebra finch mapping pedigree containing 354 individuals was genotyped at these 26 loci (Table 1). Unfortunately, one of these markers (ZF01-170) failed to amplify correctly within its multiplex set.

\section{Microsatellite genotyping and error rate checking}

The majority of the pedigree individuals were of known phenotypic sex (171 females, 175 males and 8 individuals of unknown sex). All individuals were PCR sex-typed using the Z-002 sex-typing primers [54] during the genotyping to check for any identity errors. There were two cases of mismatches between genotypic and phenotypic sex; these samples, along with eleven others, contained inheritance errors, exhibiting incompatible genotypes with known relatives. They were excluded from further analysis.

When genotyping the whole pedigree, a Qiagen multiplex mix was used during PCR amplification. Four multiplex sets were designed, each containing between 6-9 markers (Table 1). All markers in each set were co-amplified and loaded onto an ABI 3730 DNA Analyzer. The PCR program was the same as used in the singleplex reactions except the annealing temperature (Ta) was increased to $57^{\circ} \mathrm{C}$; see Qiagen multiplex guidelines for the rest of the protocol.

Two markers (ZF01-196 and ZF01-025) were included in multiple multiplex sets to verify repeatability between the different PCR sets. Marker ZF01-196 was multiplexed in sets 2, 3 and 4 and ZF01-025 in sets 2 and 3. For ZF01196 the number of samples that did not amplify was significantly greater in set 2 (42) than in sets 3 (10) and 4 (18) $\left(x^{2}=23.8, p<0.005\right.$, d.f. $\left.=2\right)$. This was also the case for ZF01-025 with set 2 containing 61 ungenotyped samples compared to 24 in set $3\left(x^{2}=16.1, \mathrm{p}<0.001\right.$, d.f. $\left.=1\right)$. The lower call rate in Set 2 is probably due to the increased number of markers incorporated into this set, as increased competition between primers could lead to reduced amplification of some loci. In total, 12 of the 273 individuals, which could be genotyped at locus ZF01-196 in all 3 sets, exhibited 2 different genotypes. The genotype that was exhibited in 2 of the sets was assumed to be the correct one, which gave a $1.46 \%$ error rate at this locus. The repeatability of locus ZF01-025 was better with only one genotyping inconsistency within the 289 samples that were genotyped in both sets giving an error rate of $0.17 \%$. The errors were distributed across all multiplex sets, making it difficult to identify the reasons for these anomalies, but suggesting that they were not due to consistent differences between the quality or reliability of the multiplexes.

An alternative method of checking the error rate is to measure the frequency of deviations from Mendelian inheritance. After genotyping the full pedigree we used the programs PedCheck and CERVUS v3.0 to assess the quality of the data $[55,56]$. Where parent-offspring genotype mismatches were identified we reanalysed the 3730 output in GENEMAPPER. In most cases an allele had been missed or incorrectly genotyped and was therefore corrected. After this, CERVUS was used to estimate null allele frequencies, and the remaining parent offspring mismatches were used to estimate the error rate. For some markers with high estimated null allele frequencies it was possible to identify where null alleles were segregating in the pedigree. In order to keep as many informative recombination events as possible in the analyses, 
these null alleles were dummy-coded as allele '99' (for markers ZF01-136, ZF02-068 \& Smm4(ZF09-004)). These data could then still be used to create the linkage maps. Any markers which had a parent-offspring error rate of $>2 \%$ were excluded from further analysis (4 markers) as it has been shown by simulation that typing error can cause map inflation and affect marker allocation to linkage maps [32]. In total the number of microsatellite markers initially used to create linkage maps of chromosomes Tgu1, Tgu1A, Tgu2 and Tgu9 were 5, 4, 6 and 6 respectively (Table 1 ).

\section{Map construction}

Linkage maps were constructed using a version of CriMap 2.4 [57] modified by Xuelu Liu (Animal Genomics and Breeding group, Monsanto Company) to accommodate large numbers of markers segregating in complicated pedigree structures. Initially, the pedigree was split into 14 sub-families using the CRIGEN function. Then the TWOPOINT command was used to calculate the two-point LOD scores between all possible marker pairs. AUTOGROUP was implemented to assign the markers to linkage groups. AUTOGROUP groups markers via an iterative process based on marker variability and linkage quality, starting at an upper, stringent layer and proceeding through lower, less stringent layers. The parameter layers were as follows: Layer 1 (40, 2.0, 2, 0.9); Layer 2 (20, $1.5,3,0.7)$; Layer $3(10,1.0,5,0.6)$; Layer $4(5,0.4,6,0.5)$. The lower layer defines the minimum requirements for inclusion in a linkage group. In this way, linkage groups were created between markers that were linked with a two-point LOD score $>5$, had a minimum of 0.4 times the average number of meioses, shared linkages with no more than six other groups and had a minimum linkage ratio (i.e. the proportion of two point linkages for a given marker to other markers in the same linkage group) of 0.5 . Once the markers were assigned to linkage groups relative marker order was determined as described below.

During the initial stages of map building redundant markers were removed using the HAPLOGROUP command. This identified tightly linked markers (recombination fraction $=0$, LOD $>10$ ) and used only the most informative marker in each haplogroup for map assembly. Using the remaining informative markers, framework maps were created using the BUILD command. The BUILD command was implemented by entering two linked, informative markers with recombination fractions of between 0.1-0.2 as ordered loci, and inserting the remaining loci. The likelihood threshold was set at 5, so the marker order with a likelihood of LOD $\geq 5$ better than the next most likely order was chosen. The process was repeated twice, each time starting with a different pair of markers. Of the three maps, the one with the most markers was chosen as the first framework map. The FLIPS5 function was used to shuffle marker orders and identify the most likely marker order. Using this framework map, we added the remaining markers by progressively dropping the likelihood threshold to $\mathrm{LOD}=3,1,0.1,0.01$ and then 0.001 . This results in a comprehensive map of all markers, with the final order determined by the best likelihood given the data available. The positions of markers mapped at the lower LOD thresholds are less well supported. In most studies a LOD of $\geq 3$, i.e. marker order 1000 times more likely than any other marker order is considered a conservative (or framework) map [58]. The FLIPS5 command was again used to check that no alternative order had a higher likelihood. Next, the markers previously excluded (via the HAPLOGROUP command) were added to the assembled map using BUILD at a likelihood threshold of LOD > 0.001 and marker order was again rechecked using FLIPS5. Finally the command CHROMPIC was used to identify any putative double recombination events between closely linked markers that may be indicative of incorrect marker order or genotype errors. CHROMPIC provides a schematic of the paternally and maternally inherited chromosomes of each individual, with the inferred grandparental origin of each marker allele indicated. This schematic shows each recombination event and flags any alleles which do not share the same grandparental origin with alleles at adjacent markers on the same haplotype. If one marker is flagged in a large number of individuals this suggests that it is either placed in the wrong location on the linkage map or has a high error rate.

Using CHROMPIC, one microsatellite marker (ZF01054) was excluded from Tgu1A as even when positioned in its location with highest likelihood it was consistently flagged, suggesting it had a high error rate. This was also supported by the fact that its presence increased map length by a substantial margin $(20 \mathrm{cM})$ [32]. A subsequent BLAST of the zebra finch trace file used for the primer design against the released zebra finch genome sequence, revealed that the first two bases at the 5 ' end of the forward primer were not consistent with the genome assembly sequence. This could potentially cause allelic drop-out leading to the high degree of error that is likely present in the genotypes at this locus. This exclusion reduced the number of microsatellites on this linkage group to three. All linkage map distances are expressed in Kosambi centiMorgans (cM) and the map figures were constructed in MapChart [59].

\section{Examining map accuracy}

Linkage maps were constructed for the microsatellites and the SNPs separately using the approach outlined above. We also built combined linkage maps including both the microsatellites and SNPs. We constructed these combined maps via two different methods. The first 
approach followed the conventional method as described above and elsewhere [40]. Typically, this would mean microsatellites would be among the first markers added to the map, as they are highly informative. Hereafter the map is termed the 'SNPs \& microsatellites conventional' map. The second approach involved adding the relevant microsatellites directly to the comprehensive SNP linkage maps (presented in [40]). This was carried out using BUILD, and as with previous approaches the likelihood threshold was progressively reduced $(\mathrm{LOD} \geq 5,3,1,0$, $0.05,0.001)$ until all the microsatellites were added to the linkage map. Here the microsatellites were added after the SNPs (hereafter this is termed the 'SNPs preceding microsatellites' map).

To validate the accuracy of the maps we compared marker order on each linkage map with the order on the zebra finch genome assembly http://genome.wustl.edu/ genomes/view/taeniopygia guttata/. We also compared SNP-only maps with maps that contained SNPs and microsatellites, in order to test whether the inclusion of microsatellites caused map length inflation.

\section{Results \& Discussion \\ Error rate}

Microsatellite genotyping error has been highlighted as a major cause for concern in population genetic analyses, including parentage assignment (reviewed in [27]). Particular attention has been given to studies using noninvasive techniques for DNA acquisition, but errors are present in all studies and this is increasingly being recognised in the literature [60]. Even in studies dealing with high quality DNA samples, the potential for error remains, particularly those caused by human error. When one investigation critically assessed the degree of genotyping error in an Antarctic fur seal paternity exclusion study, up to $93 \%$ of the errors were attributable to human mistakes [26,27].

The greater number of alleles of microsatellite markers, along with a less automated method of genotyping leads to a higher error rate relative to SNPs [25]. Within the SNP mapping project [40] one individual was repeatedly genotyped 7 times at all 1048 SNPs to measure repeatability, and no errors were detected (100\% accuracy). Also, across all SNPs the average parent-offspring mismatch rate was $0.17 \%$. In contrast, for the 25 microsatellites initially genotyped in the zebra finch mapping pedigree the mean error rate per locus was $1.32 \%$, based on the frequency of departures from Mendelian inheritance implemented in CERVUS (Table 1). This value is $\sim 7$ times more than the SNP error rate. For construction of the maps, only microsatellite markers with an error rate $<2 \%$ based on parent-offspring mismatches were used. As a result, four markers (ZF01-139, ZF01-180, ZF01-081 \& ZF02-038) were excluded from further analyses. For the microsatellites used in the final linkage maps the average error rate was $0.34 \%$ based on Mendelian inheritance inconsistencies. This is fairly low, although it is still almost double that found in the SNPs, even though the most error prone microsatellites have been removed from the dataset while all SNPs were retained.

Although genotyping errors are not uncommon, most can be easily identified. In this study the pedigree was known, and due to the number of markers typed in the entire pedigree (25), it was possible to detect any allele calls that caused departures from Mendelian inheritance. Any individuals involved in a parent-offspring mismatch were re-examined on the program GENEMAPPER. Eleven individuals were inconsistent with Mendelian inheritance at greater than 5 microsatellite loci and therefore were assumed to have been contaminated or incorrectly labelled. The results from these samples were subsequently excluded from all analysis. The high variability of microsatellites aids error detection as most errors will result in Mendelian incompatibilities. However, with SNPs this error-checking procedure will be less successful as they are only biallelic. Some studies have predicted that up to $30 \%$ of errors in SNP genotyping will remain undetected because they will not cause Mendelian inheritance incompatibilities $[61,62]$.

After re-examining the allele calling any remaining mismatches could be due to allelic dropout, scoring error, more cryptic biochemical artefacts or de novo mutations in the germline. Given that we have excluded loci with high error rates, identified mis-labelled or mis-pedigreed individuals and re-scored individuals that cause mismatches or unlikely double recombination events, it seems reasonable to assume that the subsequent impact of typing error on map construction that we report is conservative. Of course, it should be regarded good practice to remove as much of the detectable error as possible. It is important to note, and perhaps underappreciated, that some genotype errors will not cause Mendelian inconsistencies, but can still lead to the inference of spurious recombination events. For example, suppose an offspring with a true genotype of A/A was miss-scored as A/ $B$. If it has parents with genotype A/A (mother) and A/B (father) both the true and incorrect genotype are compatible with Mendelian inheritance. However it would be wrongly assumed that allele B was inherited from the father, which would lead to the inference of at least one, but more likely two, additional recombination events. Therefore, even with careful error checking, loci with high error rates are likely to cause map inflation.

\section{Robustness and reliability of maps (i) Assignment of markers to the correct chromosome}

The linkage maps generated by the 4 different methods are presented in additional file 2 : Table $\mathrm{S} 2$, along with the 
physical positions of each marker on the zebra finch genome assembly. As anticipated, both SNP and microsatellite markers formed linkage groups corresponding to their predicted chromosome based on the zebra finch assembly. We have only presented the sex-averaged linkage maps as there is little evidence of heterochiasmy in the SNP linkage maps [40].

The greatest difference between the SNP and microsatellite maps is the number of markers on each, with all chromosomes containing substantially more SNPs. The linkage groups Tgu1, Tgu1A, Tgu2 and Tgu 9 contain 73, 71,82 and 24 SNPs respectively compared to only 3, 5, 6 and 6 microsatellites. However, every microsatellite was assigned to the correct linkage group, even on the lowdensity microsatellite only maps. The ability to detect linkage between the microsatellites when so few are used is due to the relatively high number of informative meioses of these markers (Figure 1). Across all 265 markers, the number of phase known informative meioses was significantly greater for the microsatellites $($ mean $=216.3)$ than for the SNPs $($ mean $=62.3 ; \mathrm{t}=-10.71, \mathrm{p}<0.0001$ (two-tailed), d.f. $=19.44$ ).

The number of markers used in linkage maps obviously impacts on the coverage that the markers provide over the chromosomes. In all cases the SNPs provide a higher coverage of the 4 focal chromosomes. For linkage groups Tgu1, Tgu1A, Tgu2 and Tgu9, about 76\%, 96\%, 73\% and $90 \%$ respectively of the chromosomes are estimated to be within $2.5 \mathrm{Mbp}$ of a SNP marker. However, the coverage for the same chromosomes is only $24 \%, 20 \%, 16 \%$ and $76 \%$ for the microsatellite markers.

\section{(ii) Estimation of recombination rates and distribution of recombination events}

As previously highlighted [40,63] and observed to a degree on the framework SNP maps in Figure 2, recombination rates in the zebra finch appear greatest near the telomeres of the chromosomes, especially on the macrochromosomes. Most of the microsatellite markers were not positioned near to the end of the chromosomes and so are predicted to be in regions with lower recombination rates. Therefore, to compare recombination rates between the SNP and microsatellite maps, it was necessary to restrict comparisons to regions on the SNP map that overlap the chromosomal regions covered by the microsatellites.

The microsatellite maps consistently estimated higher recombination rates than did the SNP maps. For example, on Tgu1A the microsatellites span a predicted 24.4 Mbp and the linkage distance is $15.8 \mathrm{cM}$, whereas a similar region covering $21.7 \mathrm{Mbp}$ is only $0.6 \mathrm{cM}$ on the SNP map. On linkage group Tgu1 (region spanning $~ 31.4 \mathrm{Mbp}$ to $94.5 \mathrm{Mbp})$ the microsatellite map exhibits a recombination rate $\left(0.143 \mathrm{cM} \mathrm{Mb}^{-1}\right)$ more than 7 times greater than the SNP map (0.02 $\left.\mathrm{cM} \mathrm{Mb}^{-1}\right)$. The Tgu2 maps are more difficult to compare as both comprehensive maps exhibit rearrangements compared to the genome sequence (Additional file 2: Table S2). For these we used the distal microsatellites according to the physical chromosome positions and used the whole of the genetic map length to estimate the recombination rate. As the marker order with the highest likelihood is typically shorter than any alternative order this should give a conservative estimate. The microsatellite recombination rate $\left(0.24 \mathrm{cM} \mathrm{Mb}^{-1}\right)$ was ten times greater than that predicted by the SNP map $\left(0.02 \mathrm{cM} \mathrm{Mb}^{-1}\right)$. This degree of inflation is worrying, especially considering the attempts to control for the influence of microsatellite typing error. For Tgu9 we also estimated the recombination rates using the distal microsatellites according to the genome assembly. The maps lengths were more similar but the recombination rate of the microsatellite maps was still inflated (microsatellite map $=2.22 \mathrm{cM} \mathrm{Mb}^{-1}$, SNP map $\left.=1.75 \mathrm{cM} \mathrm{Mb}^{-1}\right)$. In general, the difference between SNP and microsatellite recombination rates seems to be most pronounced in the central parts of macro-chromosomes. This is logical, as recombination in these regions is relatively rare, and therefore the relative impact of spurious recombination events caused by typing error will be greater than in regions where true recombination events are more frequent.

The degree of map inflation caused by microsatellites was reduced, but still noticeable, when microsatellites

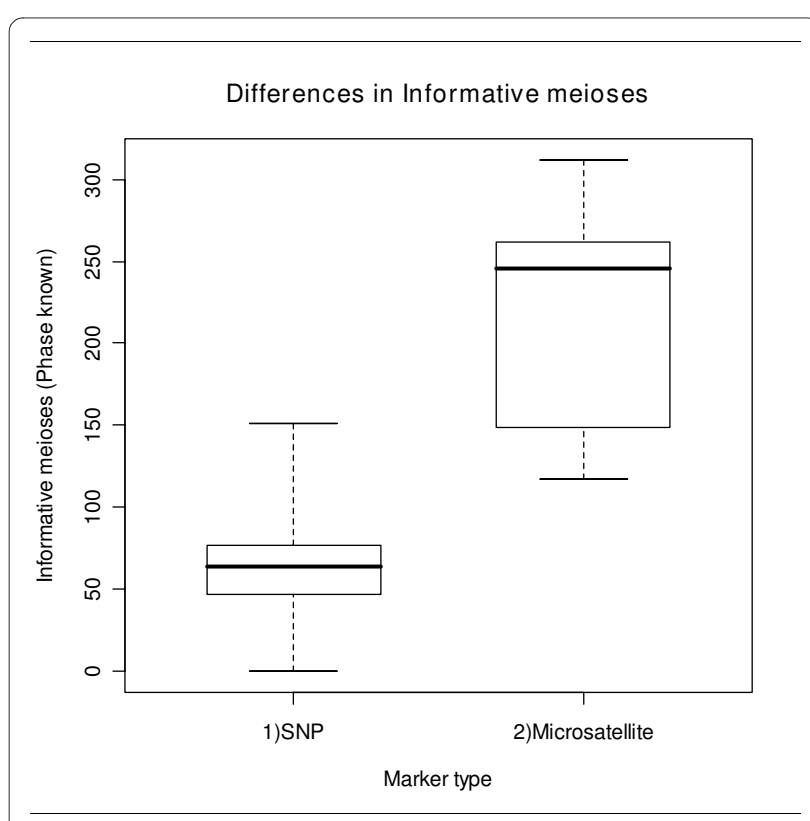

Figure 1 Number of informative meioses. Comparison of the number of informative meioses of microsatellites and SNPs. Informative meioses are highly dependent on the variability of the marker. The microsatellite markers exhibit significantly more informative meioses than the SNPs (t-test, $t=10.71, p<0.0001$ (two-tailed), d.f. $=19.44$ ). 
and SNPs were combined via the conventional build method. In general, maps built from microsatellites and SNPs were longer than SNP-only maps, even though the microsatellites were only distal to the SNPs in the Tgu9 map (Figure 3). Tgu1: SNP map = $63.7 \mathrm{cM}$; SNP and microsatellite map $=89.2 \mathrm{cM}$; Tgu1A: SNP map $=63.3 \mathrm{cM}$, SNP and microsatellite map $=83.2 \mathrm{cM}$; Tgu 2: SNP map $=$ $34.7 \mathrm{cM}, \mathrm{SNP}$ and microsatellite map $=64.6 \mathrm{cM}$; Tgu 9: SNP map $=60.5 \mathrm{cM}, \mathrm{SNP}$ and microsatellite map $=79.4$ $\mathrm{cM}$.

One conceivable explanation for the differences between the microsatellite and SNP maps is that the proportion of missing data was three times greater for microsatellties (15\%) than SNPs (5\%). This is unlikely to lead to map inflation of the microsatellite maps as there is no reason why missing genotypes should be biased with respect to whether they affect recombinant or nonrecombinant meiotic events. To test this further we simu- lated 10 replicates of the LG1 SNP map $(63.7 \mathrm{cM})$ with $15 \%$ missing data and then rebuilt the maps. The simulated datasets had a mean map length almost identical to the original (mean length $=64.1 \mathrm{cM}$; SE $0.9 \mathrm{cM}$ ). In other words there was no evidence that map inflation of the microsatellite maps was caused by a higher genotyping failure rate. It is also worth noting that the greater variability of microsatellites means that they resulted in a much higher number of informative meioses than the SNPs (see Figure 1), which more than offsets the higher failure rate.

The most likely explanation for the discrepancies in the map lengths is the higher typing error rate of the microsatellites. It seems even after carefully checking for errors and excluding error prone markers, recombination rate appears inflated. This could be a phenomenon in many microsatellite based linkage maps and needs to be considered when comparing linkage maps constructed from dif-

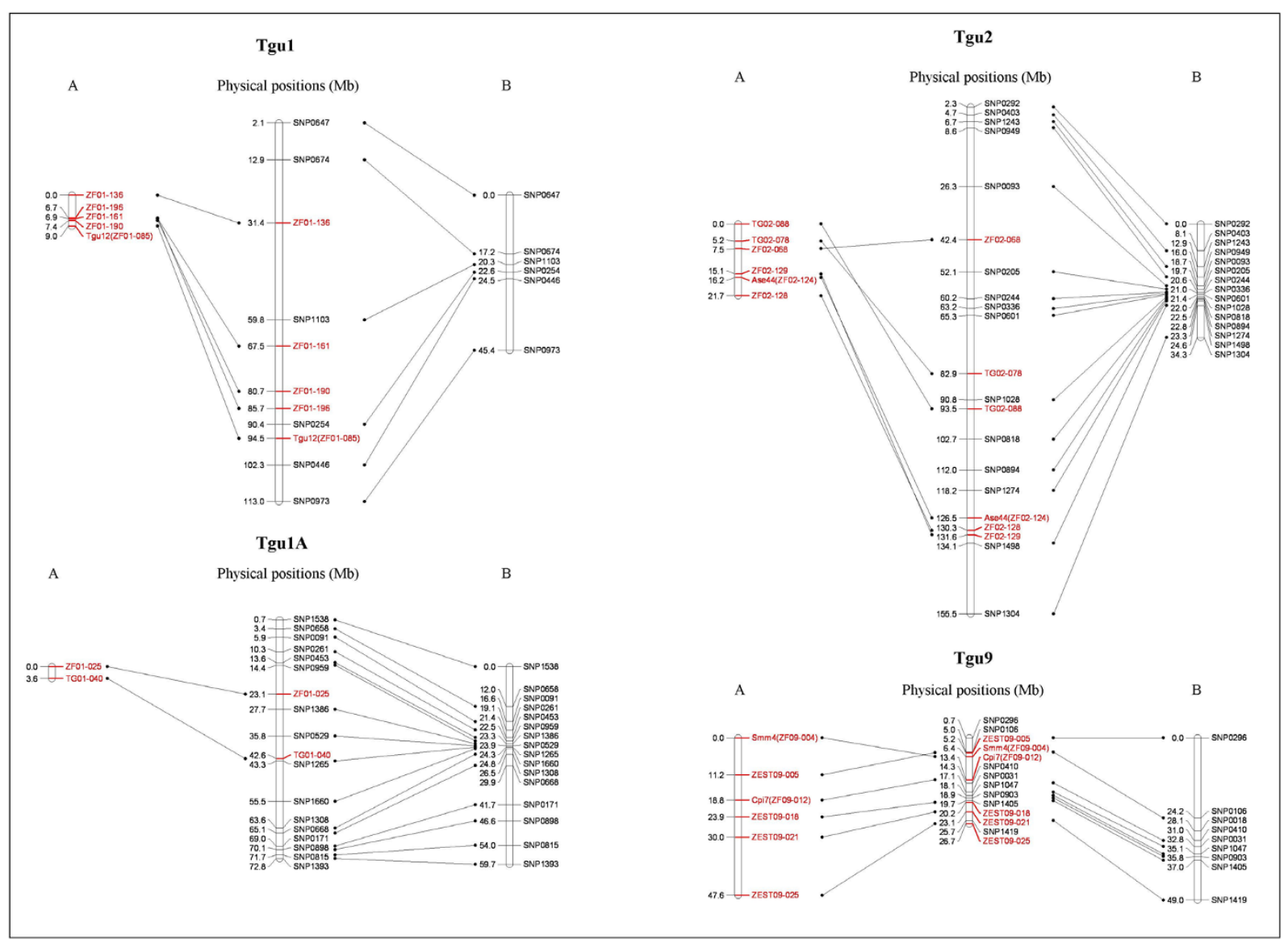

Figure 2 Separate SNP and microsatellite framework maps. Linkage maps built via the conventional method show that (A) marker order of microsatellites maps does not always correspond to the physical order on the zebra finch genome assembly (centre). However, for all four chromosomes the order of maps built with only the SNP markers (B) does agree with the zebra finch assembly order. Framework maps with marker order supported by $L O D \geq 3$ are presented. Microsatellites are highlighted in red and linkage map positions are given in CM (A and B). The predicted genome assembly positions for the markers (centre) are given in $\mathrm{Mb}$. 
ferent types of marker. Recently, a similar conclusion was reached in a paper that combined SNP, microsatellite and AFLP data to build a linkage map for the chicken genome [23]. During construction of this high density linkage map, $70 \%$ of the previously mapped markers (the majority of which were microsatellites) had to be excluded from the updated map (which mostly contained SNPs), as they caused map inflation, despite rigorous error checking when the microsatellite map was first constructed [24]. This phenomenon could be prevalent for many previously created microsatellite linkage maps and it seems likely that subsequent high density SNP maps will reveal many medium density microsatellite maps to be inflated.

\section{(iii) Accuracy of marker order}

To validate the marker order of SNP and microsatellite maps, the zebra finch genome sequence assembly was used as a template against which all linkage maps were evaluated. It is important to note that the SNP map has been used as a reference during the latter part of the zebra finch sequence assembly process (for a more detailed account of the zebra finch assembly process see http://genome.wustl.edu/pub/organism/

Other Vertebrates/Taeniopygia guttata/assembly/

Taeniopygia guttata-3.2.4/). However, the degree to which this has influenced the order of markers on the physical map is thought to be negligible. As a precaution we have also used assembled supercontigs as a reference against which to compare orders of the different linkage maps (see below). These supercontigs were created independently of any linkage map and so provide a robust test of the accuracy of map order, without any potential influence of the linkage map on the assembled zebra finch sequence.

Very few of the comprehensive maps are in complete agreement with the assembled genome sequence. It is only the microsatellite map for Tgu1A and the SNP map for Tgu9 that adhere completely to the sequence order. This is not surprising, as by definition, the comprehensive map may have a marker order with only marginally stronger statistical support than the next most likely order. Marker orders supported by a LOD $<3.0$ are not generally regarded as definitive. Therefore, it is more revealing to examine framework maps where the best order is supported by a LOD $\geq 3$ and is assumed to be reliable. Here all the SNP maps are in complete agreement with the genome assembly, but the order of three of the four microsatellite maps differs from the genome assembly (see Figure 2); the exception is Tgu1A, which only includes 2 markers. This would indicate that even the most conservative microsatellite maps are unreliable. For example, ZF09-004, (estimated null frequency = 0.049 , estimated error rate $=0.018$ ) causes an apparent inversion (relative to the physical assembly) on linkage group Tgu9 which is not supported by the SNPs. Even after exclusion of errors and selection of markers with less than $2 \%$ error rates, the microsatellite linkage maps are not only inflated, but also exhibit different marker orders to the known sequence.

In general, the SNPs-only and 'SNPs preceding microsatellites' maps provide a closer correspondence to the genome assembly than the microsatellites-only and the 'SNPs \& microsatellites conventional' maps (Additional file 2: Table S2). Examination of the framework maps (marker order supported by a LOD $\geq 3$ ) reveals that the 'SNPs preceding microsatellite' (Additional file 2: Table S2) framework maps correspond to the genome assembly for every chromosome, whereas the microsatellite-only and the 'SNPs and microsatellites conventional' framework maps generally do not (Additional file 2: Table S2). The reason for these discrepancies is likely to be typing error or null alleles at the microsatellites, which have previously been found to inflate linkage maps and support incorrect marker orders [32].

It could be argued that because the SNP map was used to help assemble the zebra finch genome its errors could have been incorporated into the physical assembly, thereby making the assembly an inappropriate reference against which to compare the microsatellite and SNP maps. To address this issue we compared the order of markers in the "SNP only" and "Microsatellite only" maps against the supercontig sequences which were created independently of the linkage maps. These are assembled genome sequences $(\mathrm{n}=37,698)$ with an average length of $\sim 10 \mathrm{Mb}$. For each framework marker (Figure 2) the supercontig location (both the supercontig name and position within that supercontig) was obtained via a BLAST search of the zebra finch genome. Any supercontigs that contained either $\geq 3$ framework SNPs (4 contigs) or microsatellites ( 1 contig) were used to investigate map accuracy by comparing the order of markers on the linkage maps and supercontigs. For all supercontigs containing $\geq 3$ framework SNPs (Contig22 $=5$ SNPs, Contig $2=4$ SNPs, Contig43 $=3$ SNPs and Contig41 $=3$ SNPs) the marker order was identical to the "SNP only" linkage map. However, the 3 framework microsatellites with BLAST positions on Contig 2, were ordered Ase44 - ZF02-128 ZF02-129 on the supercontig and ZF02-129 - Ase44 ZF02-128 on the "Microsatellite only" linkage map. This further demonstrates the inability of the microsatellite maps to infer the correct order of markers.

It should be acknowledged that some discrepancies between the linkage maps and the physical genome sequence could be indicative of mistakes in the assembly. Genome assemblies are an ongoing process, and it is likely that the zebra finch assembly will be improved as more information becomes available, and possibly as the chicken genome assembly improves. Limitations of the zebra finch sequence assembly were apparent when per- 
A

B

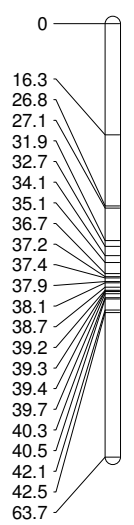

Tgu1A

A

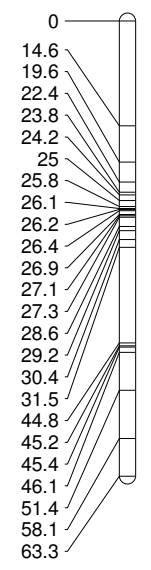

B

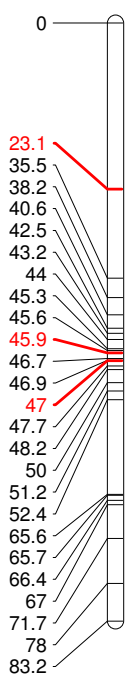

A

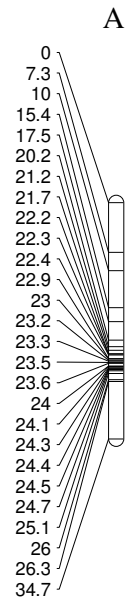

A

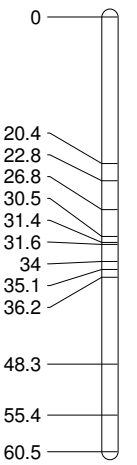

A

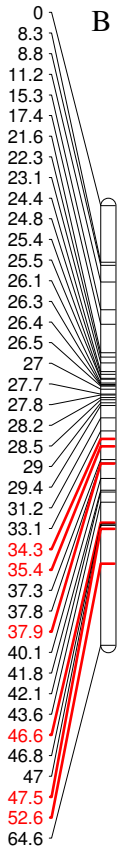

Tgu9
B
B

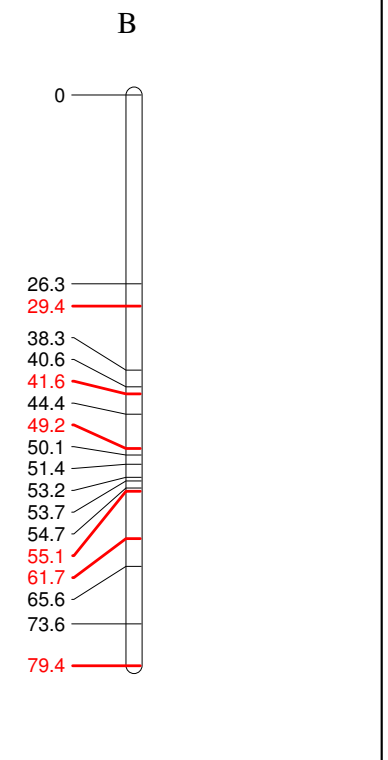

Figure 3 Inflation caused by adding microsatellites. Linkage maps created with (A) only the SNP markers are substantially shorter in length than (B) linkage maps created using both SNP and microsatellite markers when the conventional build method is used. This inflation is exhibited in all four chromosomes. Generally, the microsatellites (highlighted in red) are not distal to the SNP markers so the cause of the inflation is likely to be genotyping error at the microsatellite markers. 
forming BLAST searches; four SNP markers (SNP0444, SNP0154, SNP0853 \& SNP0018) had hits on the chicken genome assembly to the chromosomes predicted by the linkage maps, yet they fail to produce a hit on the zebra finch genome sequence. As these SNPs have been isolated and obtained from zebra finch DNA, it suggests that the zebra finch genome assembly remains relatively incomplete. Putative assembly errors however, cannot explain the much greater recombination rates of the microsatellite maps, and there is no a priori reason to expect inaccuracies in the assembly to affect marker order of microsatellites but not SNPs.

Another key point to consider when assessing these maps is that the greater marker density of the SNP maps, make the detection of 'unusual' double recombinants indicative of typing error more obvious. The problems associated with microsatellites highlighted in this paper may be less pronounced if more markers were typed, although they are unlikely to disappear completely as evidenced by the map inflation of the high density combined SNP and microsatellite maps, and by the previously undetected map inflation observed on the high density microsatellite-based chicken linkage maps [23].

\section{(iv) Comparisons of the 'SNPs and microsatellites}

\section{conventional' and 'SNPs preceding microsatellites' maps}

When combined, the microsatellites and SNPs were assigned to the predicted linkage groups based on their known chicken and zebra finch assembly positions and their earlier linkage map positions [40]. Interestingly, the two different methods used for building maps gave quite disparate map lengths (Figure 4, Additional file 2: Table S2). The linkage maps built via the conventional method, which typically means microsatellites are among the earliest to be added to the map, were longer than the equivalent maps that were constructed by adding microsatellites to the SNP maps (Tgu1 $11.8 \mathrm{cM}$ longer, Tgu1A $0.9 \mathrm{cM}$ longer, Tgu2 $10.2 \mathrm{cM}$ longer, Tgu9 $1.0 \mathrm{cM}$ longer). Furthermore, maps built by the conventional method had marker orders supported by a lower likelihood (Tgu1 35.5 lower, Tgu1A 7.5 lower, Tgu2 87.0 lower, Tgu9 9.6 lower) and showed more inconsistencies with the zebra finch assembly order, suggesting that the conventional method was more error prone than when mapping the SNPs first (Figure 4, Additional file 2: Table S2).

It is likely that the combination of greater informativeness and higher errors of the microsatellites cause them to be added first but in the wrong order. Subsequent addition of the SNPs fails to rectify the errors as the relatively large number of SNPs (compared to microsatellites) renders the FLIPS5 command unable to resolve errors in marker order between microsatellites separated by large numbers of SNPs. FLIPS takes consecutive markers, reorders them and produces likelihood scores for these different orders. Due to computational constraints it is impractical to use FLIPS for greater than five markers. What can happen in this situation is that a local likelihood maxima is reached between a group of closely linked markers such that no changes in the order of these will give a better likelihood. However, if it was possible to flip the order of more than 5 markers then a more accurate order may be revealed.

One of the outcomes of this investigation is that the conventional method of building linkage maps has to be modified when using markers that differ significantly in their number of informative meioses and error rates. Markers with the lowest error rates should be included first to provide an accurate foundation, to which other markers can then be added. This may be especially important for larger chromosomes as Tgu1, Tgu1A and Tgu2 exhibited the largest differences between the two construction methods, whereas the smaller chromosome Tgu9 did not differ as substantially. It is possible that those regions of macro-chromosomes with very low recombination rates are most sensitive to typing error. However this could also be due to the markers on Tgu9 exhibiting lower error rates, and investigation of additional smaller chromosomes would be needed to confirm this observation.

\section{Conclusions}

This study highlights potential problems, as well as some solutions, of linkage mapping construction with moderately error prone markers. Although microsatellites are informative, they can provide misleading results because they have greater error rates than SNPs. Even with the usual methods of error reduction and detection in microsatellite genotyping there is still potential for map inflation and incorrect marker orders. The results of this study emphasise the importance of careful examination of CHROMPIC outputs to identify possible genotype errors, which can create spurious double recombinants. We also suggest that the least error prone markers (SNPs) should be mapped before adding the microsatellites, at least until it is computationally practical to FLIP greater than 5 markers at a time.

This study has highlighted the importance of knowing how much effect error rates have had on the final outcome of a linkage map. With map inflation likely to be present in other microsatellite-based studies, it remains to be seen whether subsequent SNP mapping projects will reveal previously undetected errors in map order or length. However, although microsatellites are more error prone than SNPs, their widespread availability, ease of typing, high polymorphism and their cross-species utility will ensure that they remain useful in linkage-mapping studies, especially for comparing the genomes of nonmodel organisms. In summary, we are not suggesting that microsatellites should be abandoned in mapping studies, 


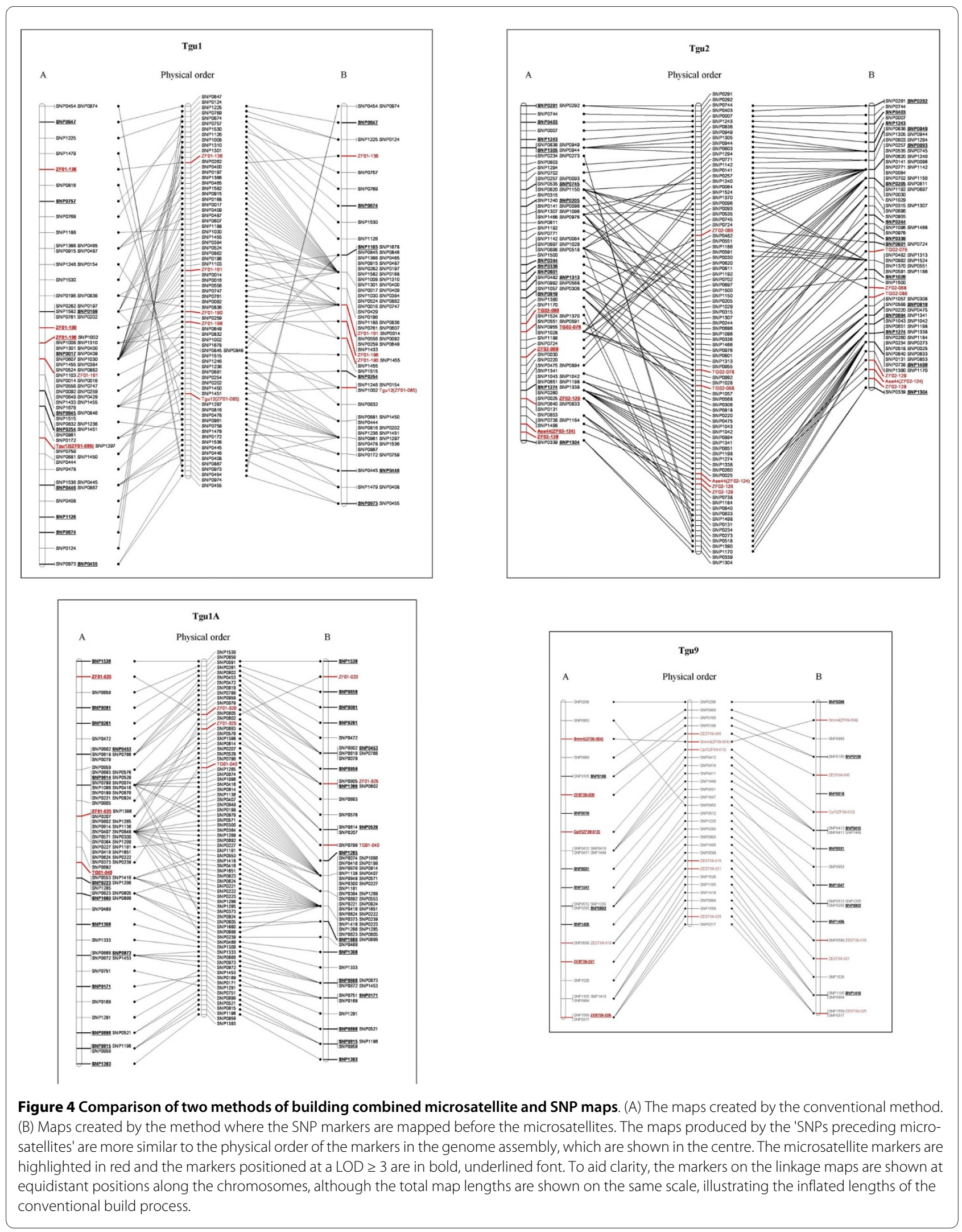


but we do urge that appropriate error checking precautions are employed.

\section{Additional material}

Additional file 1 Table S1. Characterisation of microsatellite markers. Characterisation of 26 zebra finch (Taeniopygia guttata) microsatellite markers used to create the linkage maps for the zebra finch chromosomes 1, 1A, 2, and 9.T $\mathrm{T}_{\mathrm{m}}$, melting temperature (calculated using PRIMER3)EST, expressed sequence tagt, all primer sequences were identical to zebra finch sequence (obtained either via trace files or assembled genomic sequences)a, Excluded after CHROMPIC revealed an excess of double recombination events with adjacent markers, indicative of high error rate.b, Excluded from linkage maps as parent-offspring mismatches estimated the error rate $>$ 0.02c, Could not be accurately scored after multiplexing

Additional file $\mathbf{2}$ Table S2. Results of linkage mapping. Summary of the linkage map results for four zebra finch (Taeniopygia guttata) chromosomes (Tgu1, Tgu1A, Tgu2 and Tgu9). The predicted positions of all markers on the zebra finch genome sequence after a BLAST analysis are included. Linkage map positions highlighted in bold are the framework loci that are supported at a LOD score $\geq 3$ for that linkage map.

\section{Authors' contributions}

$A B$ participated in the microsatellite discovery, carried out the microsatellite genotyping and linkage map construction and drafted the manuscript. JStapley participated in the SNP linkage map construction, provided the SNP genotyping and co-drafted the manuscript. DAD participated in the microsatellite discovery and primer design. TRB developed and managed the zebra finch mapping pedigree. TB participated in the conception and design of the study. JSlate conceived of the study and participated in its design and coordination, as well as co-drafting the manuscript. All authors read and approved the final manuscript.

\section{Acknowledgements}

We thank Jayne Pellatt, Phil Young and Lynsey Gregory for their expert technical assistance in maintaining the zebra finch population. We thank Andy Krupa for extracting the genomic DNA and providing genotyping advice. Matt Hale supplied invaluable tuition in the application of the software package CriMap. We are grateful to Gavin Hinten and Matt Hale for helpful discussion. We thank two anonymous referees for their constructive suggestions. This research was supported by the United Kingdom Biotechnology and Biological Sciences Research Council (BBSRC) under grant no. BBE0175091.

\section{Author Details}

Department of Animal and Plant Sciences, University of Sheffield, Sheffield, S10 2TN, UK

Received: 6 July 2009 Accepted: 1 April 2010

Published: 1 April 2010

\section{References}

1. Sturtevant $\mathrm{AH}$ : Linear arrangement of sex-linked factors in Drosophila, as shown by their mode of association. Journal of Experimental Zoology 1913, 14:43-59.

2. Dawson DA, Akesson M, Burke T, Pemberton JM, Slate J, Hansson B: Gene order and recombination rate in homologous chromosome regions of the chicken and a passerine bird. Molecular Biology and Evolution 2007, 24(7):1537-1552.

3. Backstrom N, Brandstrom M, Gustafsson L, Qvarnstrom A, Cheng $H$, Ellegren $\mathrm{H}$ : Genetic mapping in a natural population of collared flycatchers (Ficedula albicollis): Conserved synteny but gene order rearrangements on the avian Z chromosome. Genetics 2006, 174(1):377-386

4. Dirlewanger E, Graziano E, Joobeur T, Garriga-Caldere F, Cosson P, Howad W, Arus P: Comparative mapping and marker-assisted selection in Rosaceae fruit crops. Proceedings of the National Academy of Sciences of the United States of America 2004, 101(26):9891-9896.
5. Beraldi D, Mcrae AF, Gratten J, Slate J, Visscher PM, Pemberton JM: Mapping quantitative trait loci underlying fitness-related traits in a free-living sheep population. Evolution 2007, 61(6):1403-1416.

6. Minvielle F, Kayang BB, Inoue-Murayama M, Miwa M, Vignal A, Gourichon D, Neau A, Monvoisin JL, Ito S: Search for QTL affecting the shape of the egg laying curve of the Japanese quail. Bmc Genetics 2006, 7:26.

7. Parsons YM, Shaw KL: Mapping unexplored genomes: A genetic linkage map of the Hawaiian cricket Laupala. Genetics 2002, 162(3):1275-1282.

8. Slate J: Quantitative trait locus mapping in natural populations: progress, caveats and future directions. Molecular Ecology 2005, 14(2):363-379

9. Stemshorn KC, Nolte AW, Tautz D: A genetic map of Cottus gobio (Pisces, Teleostei) based on microsatellites can be linked to the physical map of Tetraodon nigroviridis. Journal of Evolutionary Biology 2005, 18(6):1619-1624.

10. Primmer $\mathrm{CR}$, Moller AP, Ellegren $\mathrm{H}$ : A wide-range survey of cross-species microsatellite amplification in birds. Molecular Ecology 1996, 5(3):365-378

11. Davis CS, Strobeck C: Isolation, variability, and cross-species amplification of polymorphic microsatellite loci in the family Mustelidae. Molecular Ecology 1998, 7(12):1776-1778.

12. Dawson DA, Hanotte O, Greig C, Stewart IRK, Burke T: Polymorphic microsatellites in the blue tit Parus caeruleus and their cross-species utility in 20 songbird families. Molecular Ecology 2000, 9(11):1941-1944.

13. Dawson DA, Hunter FM, Pandhal J, Buckland R, Parham A, Jones IL, Bradshaw M, Jehle R, Burke T: Assessment of 17 new whiskered auklet (Aethia pygmaea) microsatellite loci in 42 seabirds identifies 5-15 polymorphic markers for each of nine Alcinae species. Molecular Ecology Notes 2005, 5(2):289-297.

14. Dib C, Faure S, Fizames C, Samson D, Drouot N, Vignal A, Millasseau P, Marc S, Hazan J, Seboun E, et al:: A comprehensive genetic map of the human genome based on 5,264 microsatellites. Nature 1996, 380(6570):152-154

15. Dietrich WF, Miller J, Steen R, Merchant MA, DamronBoles D, Husain Z, Dredge $\mathrm{R}$, Daly MJ, Ingalls KA, OConnor TJ, et al:: A comprehensive genetic map of the mouse genome (vol 380, pg 149, 1996). Nature 1996, 381(6578):172-172.

16. Bell CJ, Ecker JR: Assignment of 30 Microsatellite Loci to the Linkage Map of Arabidopsis. Genomics 1994, 19(1):137-144.

17. Roder MS, Korzun V, Wendehake K, Plaschke J, Tixier MH, Leroy P, Ganal MW: A microsatellite map of wheat. Genetics 1998, 149(4):2007-2023.

18. Bishop MD, Kappes SM, Keele JW, Stone RT, Sunden SLF, Hawkins GA, Toldo SS, Fries R, Grosz MD, Yoo JY, et al:: A Genetic-Linkage Map for Cattle. Genetics 1994, 136(2):619-639.

19. Slate J, Van Stijn TC, Anderson RM, McEwan KM, Maqbool NJ, Mathias HC, Bixley MJ, Stevens DR, Molenaar AJ, Beever JE, et al: A deer (subfamily cervinae) genetic linkage map and the evolution of ruminant Genomes. Genetics 2002, 160(4):1587-1597.

20. Hansson B, Akesson M, Slate J, Pemberton JM: Linkage mapping reveals sex-dimorphic map distances in a passerine bird. PR SOC B 2005 272(1578):2289-2298.

21. Syvanen AC: Toward genome-wide SNP genotyping. Nat Genet 2005, 37:S5-S10

22. Shen R, Fan JB, Campbell D, Chang WH, Chen J, Doucet D, Yeakley J, Bibikova M, Garcia EW, McBride C, et al:: High-throughput SNP genotyping on universal bead arrays. Mutat Res-Fund Mol M 2005, 573(1-2): 70-82

23. Groenen MAM, Wahlberg $P$, Foglio $M$, Cheng HH, Megens HJ, Crooijmans RPMA, Besnier F, Lathrop M, Muir WM, Wong GKS, et al:: A high-density SNP-based linkage map of the chicken genome reveals sequence features correlated with recombination rate. Genome Research 2009, 19(3):510-519.

24. Groenen MAM, Cheng HH, Bumstead N, Benkel BF, Briles WE, Burke T, Burt DW, Crittenden LB, Dodgson J, Hillel J, et al:: A consensus linkage map of the chicken genome. Genome Research 2000, 10(1):137-147.

25. Evans DM, Cardon LR: Guidelines for genotyping in genomewide linkage studies: Single-nucleotide-polymorphism maps versus microsatellite maps. Am J Hum Genet 2004, 75(4):687-692.

26. Hoffman J, Amos W: Microsatellite genotyping errors: detection approachesf common sources and consequences for paternal exclusion. Molecular Ecology 2005, 14(2):599-612. 
27. Pompanon F, Bonin A, Bellemain E, Taberlet P: Genotyping errors: Causes, consequences and solutions. Nat Rev Genet 2005, 6(11):847-859.

28. Slate J, Gratten J, Beraldi D, Stapley J, Hale MC, Pemberton JM: Gene mapping in the wild with SNPs: guidelines and future directions. In Genetica Springer Netherlands; 2008.

29. Bonin A, Bellemain E, Eidesen PB, Pompanon F, Brochmann C, Taberlet P: How to track and assess genotyping errors in population genetics studies. Molecular Ecology 2004, 13(11):3261-3273.

30. Taberlet $P$, Waits LP, Luikart G: Noninvasive genetic sampling: look before you leap. Trends in Ecology \& Evolution 1999, 14(8):323-327.

31. Estimated genotype error rates from bowhead whale microsatellite data [http://iwcoffice.org/ documents/sci com/SC59docs/SC-59BRG15.pdf

32. Slate J: Robustness of linkage maps in natural populations: a simulation study. PR SOC B 2008, 275(1635):695-702.

33. Hackett $C A$, Broadfoot $L B$ : Effects of genotyping errors, missing values and segregation distortion in molecular marker data on the construction of linkage maps. Heredity 2003, 90(1):33-38.

34. John S, Shephard N, Liu GY, Zeggini E, Cao MQ, Chen WW, Vasavda N, Mills T, Barton A, Hinks A, et al:: Whole-genome scan, in a complex disease, using 11,245 single-nucleotide polymorphisms: Comparison with microsatellites. Am J Hum Genet 2004, 75(1):54-64.

35. Ulgen A, Li WT: Comparing single-nucleotide polymorphism markerbased and microsatellite marker-based linkage analyses. Bmc Genetics 2005, 6(Suppl 1):S13.

36. Middleton FA, Pato MT, Gentile KL, Morley CP, Zhao X, Eisener AF, Brown A, Petryshen TL, Kirby AN, Medeiros $H$, et al:: Genomewide linkage analysis of bipolar disorder by use of a high-density single-nucleotidepolymorphism (SNP) genotyping assay: A comparison with microsatellite marker assays and finding of significant linkage to chromosome 6q22. Am J Hum Genet 2004, 74(5):886-897.

37. Xing C, Schumacher FR, Xing G, Lu Q, Wang T, Elston RC: Comparison of microsatellites, single-nucleotide polymorphisms (SNPs) and composite markers derived from SNPs in linkage analysis. Bmc Genetics 2005, 6(Suppl 1):S29.

38. Wilcox MA, Pugh EW, Zhang HP, Zhong XY, Levinson DE, Kennedys GC, Wijsman EM: Comparison of single-nucleotide polymorphisms and microsatellite markers for linkage analysis in the COGA and simulated data sets for genetic analysis workshop 14: Presentation groups 1, 2 , and 3. Genetic Epidemiology 2005, 29:S7-S28.

39. Gagneux P, Boesch C, Woodruff DS: Microsatellite scoring errors associated with noninvasive genotyping based on nuclear DNA amplified from shed hair. Molecular Ecology 1997, 6(9):861-868.

40. Stapley J, Birkhead TR, Burke T, Slate J: A linkage map of the zebra finch Taeniopygia guttata provides new insights into avian genome evolution. Genetics 2008, 179(1):651-667.

41. Nicholls JA, Double MC, Rowell DM, Magrath RD: The evolution of cooperative and pair breeding in thornbills Acanthiza (Pardalotidae). Journal of Avian Biology 2000, 31(2):165-176.

42. Dawson DA, Chittock JC, Jehle R, Whitlock A, Nogueira D, Pellatt J, Birkhead T, Burke T: Identification of 13 polymorphic microsatellite loc in the zebra finch, Taeniopygia guttata (Passeridae, Aves). Molecular Ecology Notes 2005, 5(2):298-301

43. Dawson DA, Horsburgh GJ, Kupper C, Stewart IRK, Ball AD, Durrant KL, Hansson B, Bacon I, Bird S, Klein A, et al: New methods to identify conserved microsatellite loci and develop primer sets of high utility as demonstrated for birds. Molecular Ecology Resources 2010 in press.

44. Hillier LW, Miller W, Birney E, Warren W, Hardison RC, Ponting CP, Bork P, Burt DW, Groenen MAM, Delany ME, et al:: Sequence and comparative analysis of the chicken genome provide unique perspectives on vertebrate evolution. Nature 2004, 432(7018):695-716.

45. Dawson DA, Burke T, Hansson B, Pandhal J, Hale MC, Hinten GN, Slate J: A predicted microsatellite map of the passerine genome based on chicken-passerine sequence similarity. Molecular Ecology 2006, 15(5):1299-1320.

46. Derjusheva S, Kurganova A, Habermann F, Gaginskaya E: High chromosome conservation detected by comparative chromosome painting in chicken, pigeon and passerine birds. Chromosome Research 2004, 12(7):715-723.
47. Slate J, Hale MC, Birkhead TR: Simple sequence repeats in zebra finch (Taeniopygia guttata) expressed sequence tags: a new resource for evolutionary genetic studies of passerines. Bmc Genomics 2007, 8:52.

48. Weber JL: Informativeness of Human (Dc-Da)N.(Dg-Dt)N

Polymorphisms. Genomics 1990, 7(4):524-530.

49. Brinkmann B, Klintschar M, Neuhuber F, Huhne J, Rolf B: Mutation rate in human microsatellites: Influence of the structure and length of the tandem repeat. Am J Hum Genet 1998, 62(6):1408-1415.

50. Huang $X Q$, Madan A: CAP3: A DNA sequence assembly program. Genome Research 1999, 9(9):868-877.

51. Altschul SF, Gish W, Miller W, Myers EW, Lipman DJ: Basic Local Alignment Search Tool. J Mol Biol 1990, 215(3):403-410.

52. Rozen S, Skaletsky HJ: In: Bioinformatics Methods and Protocols: Methods in molecular biology. 2000:365-386.

53. Marshall TC, Slate J, Kruuk LEB, Pemberton JM: Statistical confidence for likelihood-based paternity inference in natural populations. Molecular Ecology 1998, 7(5):639-655

54. Dawson DA: Genomic analysis of passerine birds using conserved microsatellite loci. In Phd Thesis Sheffield: University of Sheffield; 2007.

55. O'Connell JR, Weeks DE: PedCheck: A program for identification of genotype incompatibilities in linkage analysis. Am J Hum Genet 1998, 63(1):259-266.

56. Kalinowski ST, Taper ML, Marshall TC: Revising how the computer program CERVUS accommodates genotyping error increases success in paternity assignment. Molecular Ecology 2007, 16(5):1099-1106.

57. Lander ES, Green P: Construction of Multilocus Genetic-Linkage Maps in Humans. Proceedings of the National Academy of Sciences of the United States of America 1987, 84(8):2363-2367.

58. Morton NE: Significance levels in complex inheritance (vol 62, pg 690, 1998). Am J Hum Genet 1998, 63(4):1252-1252.

59. Voorips RE: MapChart: Software for the graphical presentation of linkage maps and QTLs. The Journal of Heredity 2002, 93(1):77-78.

60. Sefc KM, Payne RB, Sorenson MD: Microsatellite amplification from museum feather samples: Effects of fragment size and template concentration on genotyping errors. Auk 2003, 120(4):982-989.

61. Gordon D, Heath SC, Ott J: True pedigree errors more frequent than apparent errors for single nucleotide polymorphisms. Hum Hered 1999, 49(2):65-70.

62. Geller F, Ziegler A: Detection rates for genotyping errors in SNPs using the trio design. Hum Hered 2002, 54(3):111-117.

63. Calderon PL, Pigozzi MI: MLH1-focus mapping in birds shows equal recombination between sexes and diversity of crossover patterns. Chromosome Research 2006, 14(6):605-612.

64. Richardson DS, Jury FL, Dawson DA, Salgueiro P, Komdeur J, Burke T: Fifty Seychelles warbler (Acrocephalus sechellensis) microsatellite loci polymorphic in Sylviidae species and their cross-species amplification in other passerine birds. Molecular Ecology 2000, 9(12):2226-2231.

65. Maguire GS, Guay PJ, Mulder RA: Isolation and characterization of microsatellite markers in the southern emu-wren (Stipiturus malachurus: Aves). Molecular Ecology Notes 2006, 6(2):422-424.

66. Doerr ED: Characterization of microsatellite loci in the brown treecreeper (Climacteris picumnus) and cross-species amplification in the white-throated treecreeper (Cormobates leucophaeus). Molecular Ecology Notes 2005, 5(3):654-656.

doi: 10.1186/1471-2164-11-218

Cite this article as: Ball et al., A comparison of SNPs and microsatellites as linkage mapping markers: lessons from the zebra finch (Taeniopygia guttata) BMC Genomics 2010, 11:218 